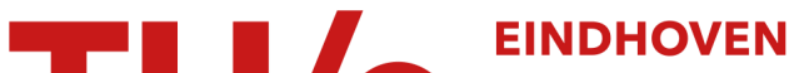 \\ UNIVERSITY OF \\ TECHNOLOGY
}

\section{Optical study of the band structure of wurtzite GaP nanowires}

Citation for published version (APA):

Assali, S., Greil, J., Zardo, I., Belabbes, A., de Moor, M. W. A., Kölling, S., Koenraad, P. M., Bechstedt, F., Bakkers, E. P. A. M., \& Haverkort, J. E. M. (2016). Optical study of the band structure of wurtzite GaP nanowires. Journal of Applied Physics, 120(4), 1-10. [044304]. https://doi.org/10.1063/1.4959147

DOI:

10.1063/1.4959147

Document status and date:

Published: 01/07/2016

\section{Document Version:}

Publisher's PDF, also known as Version of Record (includes final page, issue and volume numbers)

\section{Please check the document version of this publication:}

- A submitted manuscript is the version of the article upon submission and before peer-review. There can be important differences between the submitted version and the official published version of record. People interested in the research are advised to contact the author for the final version of the publication, or visit the $\mathrm{DOI}$ to the publisher's website.

- The final author version and the galley proof are versions of the publication after peer review.

- The final published version features the final layout of the paper including the volume, issue and page numbers.

Link to publication

\section{General rights}

Copyright and moral rights for the publications made accessible in the public portal are retained by the authors and/or other copyright owners and it is a condition of accessing publications that users recognise and abide by the legal requirements associated with these rights.

- Users may download and print one copy of any publication from the public portal for the purpose of private study or research.

- You may not further distribute the material or use it for any profit-making activity or commercial gain

- You may freely distribute the URL identifying the publication in the public portal.

If the publication is distributed under the terms of Article $25 \mathrm{fa}$ of the Dutch Copyright Act, indicated by the "Taverne" license above, please follow below link for the End User Agreement:

www.tue.nl/taverne

Take down policy

If you believe that this document breaches copyright please contact us at:

openaccess@tue.nl

providing details and we will investigate your claim. 


\section{Optical study of the band structure of wurtzite GaP nanowires}

S. Assali', J. Greil', I. Zardo', A. Belabbes', M. W. A. de Moor', S. Koelling, P. M. Koenraad, F. Bechstedt, E. P. A. M. Bakkers, and J. E. M. Haverkort

Citation: J. Appl. Phys. 120, 044304 (2016); doi: 10.1063/1.4959147

View online: http://dx.doi.org/10.1063/1.4959147

View Table of Contents: http://aip.scitation.org/toc/jap/120/4

Published by the American Institute of Physics 


\title{
Optical study of the band structure of wurtzite GaP nanowires
}

\author{
S. Assali, ${ }^{1, a), b)}$ J. Greil, ${ }^{1, c)}$ I. Zardo, ${ }^{1, d)}$ A. Belabbes, ${ }^{2, e)}$ M. W. A. de Moor, ${ }^{1, f)}$ S. Koelling, ${ }^{1}$ \\ P. M. Koenraad, ${ }^{1}$ F. Bechstedt, ${ }^{2}$ E. P. A. M. Bakkers, ${ }^{1,3}$ and J. E. M. Haverkort ${ }^{1}$ \\ ${ }^{1}$ Department of Applied Physics, Eindhoven University of Technology, $5600 \mathrm{MB}$ Eindhoven, The Netherlands \\ ${ }^{2}$ Institut für Festkörpertheorie und-optik, Friedrich-Schiller-Universitat, Max-Wien-Platz 1, D-07743 Jena, \\ Germany \\ ${ }^{3}$ Kavli Institute of Nanoscience, Delft University of Technology, 2600 GA Delft, The Netherlands
}

(Received 20 May 2016; accepted 8 July 2016; published online 25 July 2016)

\begin{abstract}
We investigated the optical properties of wurtzite (WZ) GaP nanowires by performing photoluminescence $(\mathrm{PL})$ and time-resolved PL measurements in the temperature range from $4 \mathrm{~K}$ to $300 \mathrm{~K}$, together with atom probe tomography to identify residual impurities in the nanowires. At low temperature, the $\mathrm{WZ} \mathrm{GaP} \mathrm{luminescence} \mathrm{shows} \mathrm{donor-acceptor} \mathrm{pair} \mathrm{emission} \mathrm{at} 2.115 \mathrm{eV}$ and $2.088 \mathrm{eV}$, and Burstein-Moss band-filling continuum between 2.180 and $2.253 \mathrm{eV}$, resulting in a direct band gap above $2.170 \mathrm{eV}$. Sharp exciton $\alpha-\beta-\gamma$ lines are observed at $2.140-2.164-2.252 \mathrm{eV}$, respectively, showing clear differences in lifetime, presence of phonon replicas, and temperaturedependence. The excitonic nature of those peaks is critically discussed, leading to a direct band gap of $\sim 2.190 \mathrm{eV}$ and to a resonant state associated with the $\gamma$-line $\sim 80 \mathrm{meV}$ above the $\Gamma_{8 \mathrm{C}}$ conduction band edge. Published by AIP Publishing. [http://dx.doi.org/10.1063/1.4959147]
\end{abstract}

\section{INTRODUCTION}

The electronic band structure of a semiconductor is determined by the periodicity of the crystal structure. When the atomic planes are stacked differently, the opto-electrical properties of the material will be changed. Among the indirect band gap III-V semiconductors with zinc blende (ZB) crystal structure, $\mathrm{GaP}$ is predicted to become a direct band gap material when grown with wurtzite (WZ) crystal structure. ${ }^{1-5}$ Semiconductor nanowires (NWs) have shown excellent control of the growth of ZB-WZ phases, ${ }^{6-9}$ allowing crystal structure band engineering. ${ }^{10-12}$ Recently, the Auseeded growth of high purity WZ GaP nanowires via vaporliquid-solid (VLS) method was demonstrated, and the optical properties have been investigated. ${ }^{13-18}$ Photoluminescence (PL) measurements on patterned arrays of $\mathrm{WZ} \mathrm{GaP} \mathrm{wires}$ showed optical emission at $2.09 \mathrm{eV}$ with a short lifetime of $\sim 0.8 \mathrm{~ns}$, indicating a direct band gap semiconductor. ${ }^{13}$ Strain dependence of the optical emission ${ }^{17}$ and estimation of the dielectric constant ${ }^{18}$ provide additional information on this material system. Furthermore, efficient water reduction in $\mathrm{WZ} \mathrm{GaP} \mathrm{nanowires} \mathrm{resulting} \mathrm{from} \mathrm{an} \mathrm{enhanced} \mathrm{optical}$ absorption further indicates the direct band gap nature of WZ GaP. ${ }^{19}$ However, a detailed study of the optical properties of WZ GaP and a correlation with the predicted band

\footnotetext{
a)simone.assali@polymtl.ca

b) Present address: Department of Engineering Physics, Polytechnique Montréal, C. P. 6079, Succ. Centre-Ville, Montréal, Québec H3C 3A7, Canada.

${ }^{c)}$ Present address: Institute of solid state electronics, Vienna university of technology, Floragasse 7, 1040 Vienna, Austria.

${ }^{d)}$ Present address: Department of Physics, University of Basel, Klingelbergstrasse 82, 4056 Basel, Switzerland.

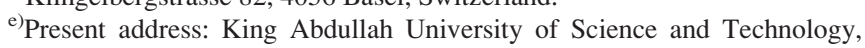
Thuwal 23955-6900, Kingdom of Saudi Arabia.

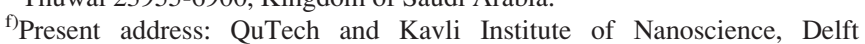
University of Technology, 2600 GA Delft, The Netherlands.
}

structure is still missing. The electronic band structure calculated for $\mathrm{WZ} \mathrm{GaP}$ with the density functional theory (DFT) simulating the excitation aspect within the local density approximation (LDA)-1/2 approximation predicts three valence bands for the heavy-hole $\left(\Gamma_{9_{\mathrm{v}}}\right)$, light-hole $\left(\Gamma_{7 \mathrm{v}+}\right)$, and split-off $\left(\Gamma_{7 \mathrm{v}-}\right)$ bands, as shown in Fig. $1(\mathrm{a}) .{ }^{3,20}$ The $\Gamma_{9 \mathrm{v}}$ and $\Gamma_{7 \mathrm{v}+}$ valence bands are non-degenerate at the $\Gamma$-point due to the crystal field. In the conduction band, the $\Gamma_{8 \mathrm{c}}$ band edge at the $\Gamma$-point is the lowest band, with a considerably lower energy than the $\Gamma_{7 \mathrm{c}}$ band, resulting in a fundamental $\Gamma_{9 \mathrm{v}}-\Gamma_{8 \mathrm{c}}$ band gap of $2.123 \mathrm{eV}$ and in transitions with the $\Gamma_{7 \mathrm{c}}$ band above $2.888 \mathrm{eV} .{ }^{20}$ This situation is fundamentally different from other III-V materials such as WZ GaAs and WZ $\mathrm{InP}$, where the minimum in the conduction band is predicted with $\Gamma_{7 \mathrm{c}}$ symmetry. ${ }^{3,5,20}$ In addition, the indirect $\mathrm{M}$ minimum of $\mathrm{WZ} \mathrm{GaP}$ is predicted at $2.233 \mathrm{eV}$; hence, the band gap is expected to be direct. The transitions between the $\Gamma_{9 \mathrm{v}}, \Gamma_{7 \mathrm{v}+}$, and $\Gamma_{7 \mathrm{v}-}$ valence bands and the $\Gamma_{8 \mathrm{c}}$ conduction band in WZ GaP are labelled as A-B-C in Fig. 1(a). A correction to the values for the band splitting in Ref. 20 results in band distances $\Delta_{\mathrm{AB}}=42.5 \mathrm{meV}$ and $\Delta_{\mathrm{BC}}=132.5 \mathrm{meV}$. At the $\Gamma$-point, only the A transition is weakly allowed due to the hexagonal crystal field for light polarized perpendicular to the $\mathrm{c}$-axis, while the $\mathrm{B}-\mathrm{C}$ transitions are completely forbidden (see Fig. 1(e)). ${ }^{21}$ The wavevector dependence of the transition matrix elements of the A-B-C transitions in WZ GaP is shown in Figs. 1(b)-1(d), ${ }^{20}$ where a non-zero transition matrix element at the $\Gamma$-point is observed only for the A transition. However, a substantial increase of the matrix element is predicted along the $\Gamma$-M direction. As a consequence, excitons bound to impurities can relax the selection rules ${ }^{22}$ since a strong localization in real space results in a considerable extension in $k$-space. This results in an average over the $k$-dependent matrix elements that leads to a non-zero transition probability, with the oscillator strength $f$ given by $f \propto\left|\Sigma_{\mathbf{k}} \mathrm{p}(\mathbf{k}) \mathrm{A}(\mathbf{k})\right|^{2},{ }^{23}$ where $p(k)$ is 
(a)

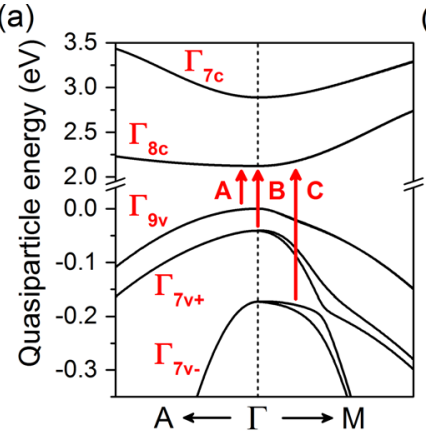

(c)

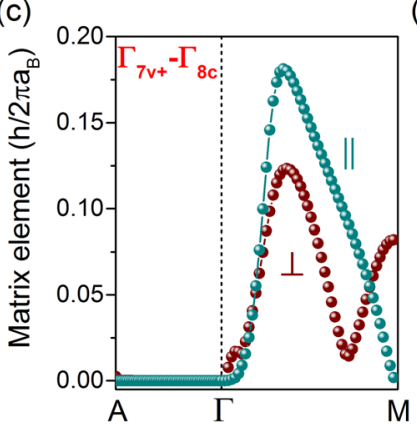

(b)

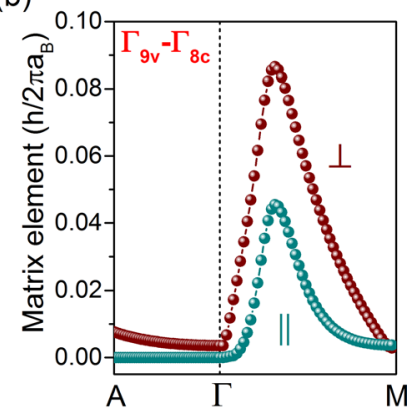

(d)

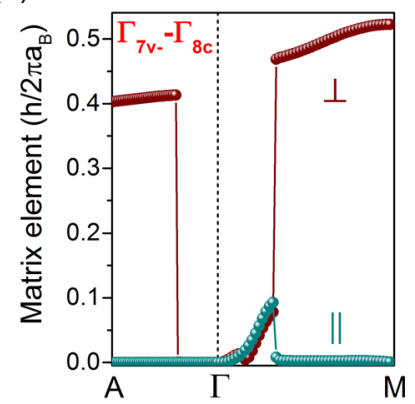

(e)

\begin{tabular}{|l|c|c|}
\hline Transition & Selection rule & Energy (eV) \\
\hline$A\left(\Gamma_{9 v-} \Gamma_{8 c}\right)$ & $\perp$ (weak) & 2.123 \\
\hline$B\left(\Gamma_{7 v+}-\Gamma_{8 c}\right)$ & forbidden & 2.166 \\
\hline$C\left(\Gamma_{7 v-}-\Gamma_{8 c}\right)$ & forbidden & 2.298 \\
\hline
\end{tabular}

FIG. 1. (a) Band structure of WZ GaP calculated with the DFT LDA-1/2 method ${ }^{20}$ with the A-B-C transitions indicated by arrows. ((b)-(d)) Plot of the transition matrix elements as a function of $k$-vector for the A-B-C transitions for the polarization perpendicular $(\perp)$ and parallel $(\|)$ to the c-axis. ${ }^{20}$ (e) Selection rules $(k=0)$ for the optical transitions with $\Gamma_{8 \mathrm{c}}{ }^{21}$ The energy values for the A-B-C transitions are estimated from DFT LDA-1/2 calculations.

the momentum matrix element. In the case of a free exciton, $A(k)$ is the Fourier transform of the envelope function. Instead, for a bound exciton, $A(k)$ can be interpreted mainly due to the envelope function of the particles bound to the point defect. A striking example is the case of an exciton bound to an iso-electronic nitrogen impurity in $\mathrm{ZB} \mathrm{GaP.}{ }^{24}$

In this work, we present power- and temperaturedependent micro-PL and time-resolved PL (TRPL) measurements on WZ GaP nanowires, combined with atom probe tomography (APT) to identify the presence of residual impurities. We show that, although the matrix element for the A transition is predicted to be very weak at $k=0$, the WZ GaP nanowires still feature a very rich photoluminescence spectrum, showing sharp peaks labeled $\alpha-\beta-\gamma$ at 2.140 $-2.164-2.252 \mathrm{eV}$. This provides a deeper insight into the WZ $\mathrm{GaP}$ material system, where material parameters, such as experimental data for fundamental gap, spin-orbit splitting, crystal field splitting, or binding energies of donor, acceptor, or exciton, are unknown.

\section{EXPERIMENTAL AND COMPUTATIONAL DETAILS}

The WZ GaP NWs are grown using the VLS growth method in a low-pressure (50 mbar) Aixtron CCS-MOVPE,

following the growth recipe described in Ref. 13 and using a growth time of $75 \mathrm{~min}$. Arrays of gold particles with $200 \mathrm{~nm}$ diameter and $1.2 \mu \mathrm{m}$ pitch are patterned on a GaP (111)B substrate via electron-beam lithography, and used as catalyst for the VLS growth. The optical properties of the nanowires are studied using micro-PL spectroscopy in the temperature range $4-300 \mathrm{~K}$. The optical data are collected in backscattering geometry using a $\mathrm{NA}=0.7$ Nikon $50 \mathrm{X} \mathrm{CR}$ objective, and a $0.30 \mathrm{~m}$ spectrometer equipped with $1200 \mathrm{~g} / \mathrm{mm}$ and holographic $2400 \mathrm{~g} / \mathrm{mm}$ gratings (500 $\mathrm{nm}$ blazing angle). A time correlated single photon counting (SPC) detector (130 ps instrumental resolution) is used for TRPL measurements and the lifetime is extracted considering a mono-exponential decay. Two different excitation sources are used: (i) for PL measurements, a frequency-doubled Titanium-Sapphire laser $(405 \mathrm{~nm}, 80 \mathrm{MHz}, 150 \mathrm{fs}$ pulse length) fiber-coupled into $300 \mu \mathrm{m}$ core multi-mode fiber, resulting in a circular excitation spot with diameter of $\sim 9 \mu \mathrm{m}$ at the sample; (ii) for TRPL measurements, a pulsed laser diode $(420 \mathrm{~nm}$, $0.5-80 \mathrm{MHz},<300 \mathrm{ps}$ pulse length), with a circular excitation spot with diameter of $\sim 1 \mu \mathrm{m}$ at the sample.

For the APT measurements, the nanowires were picked up with a Kleindiek nanomanipulator inside a FEI Nova Dual-beam and placed on an etched silicon post. APT analyses were carried out in a LEAP 4000X-HR from Cameca. The system is equipped with a laser generating picosecond pulses at a wavelength of $355 \mathrm{~nm}$. The experimental data were collected at a laser pulse rate of $100 \mathrm{kHz}$ with laser powers between 0.6 and $1.5 \mathrm{pJ}$ per pulse. During the analysis, the sample is kept at base temperature of $20 \mathrm{~K}$ in a vacuum of $2 \times 10^{-11}$ mbar.

Ab-initio calculations are performed in the framework of the $\mathrm{DFT}^{25}$ within the local density approximation (LDA) ${ }^{26}$ as implemented in the Vienna ab-initio simulation package (VASP), ${ }^{27,28}$ where the exchange-correlation (XC) functional is parameterized according to Perdew and Zunger. ${ }^{29}$ The all-electron wave functions and the pseudopotentials for the electron-ion interaction are described within the projector-augmented wave (PAW) framework. ${ }^{30}$ The KohnSham wave functions are expanded in plane waves up to an energy cutoff of $500 \mathrm{eV}$, which ensures the energy convergence within $1 \mathrm{mRy}$ per atom. Quasiparticle corrections have been taken into account to simulate the excitation effects by using the LDA-1/2 method, ${ }^{23,31}$ which allows the inclusion of spin-orbit interaction in a rather easy manner. ${ }^{20}$ We apply this approach by preparing a pd-like excitation in the electronic system of $\mathrm{GaP}^{23}$ according to Slater's transition state idea. ${ }^{32,33}$ Consequently, the eigenvalues with $\mathrm{p}$ or $\mathrm{d}$ character are corrected, mainly shifted to lower or higher energies according to their occupation. The resulting energy gaps agree well with experimental values for the zinc-blende polytypes. $^{20}$

\section{RESULTS}

Arrays of untapered WZ GaP NWs with $200 \mathrm{~nm}$ diameter and lengths up to $13 \mu \mathrm{m}$ are shown in the scanning electron micrograph (SEM) in the inset in Fig. 2(a) and in the high-resolution transmission electron micrograph (HRTEM) 
(a)

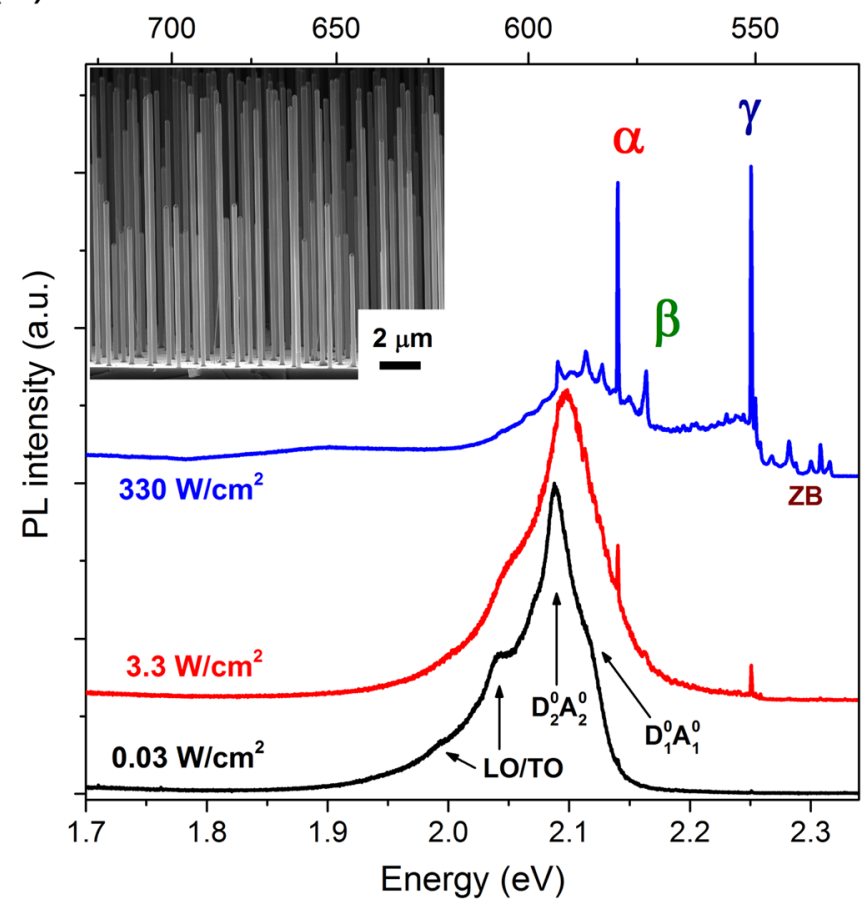

(b)

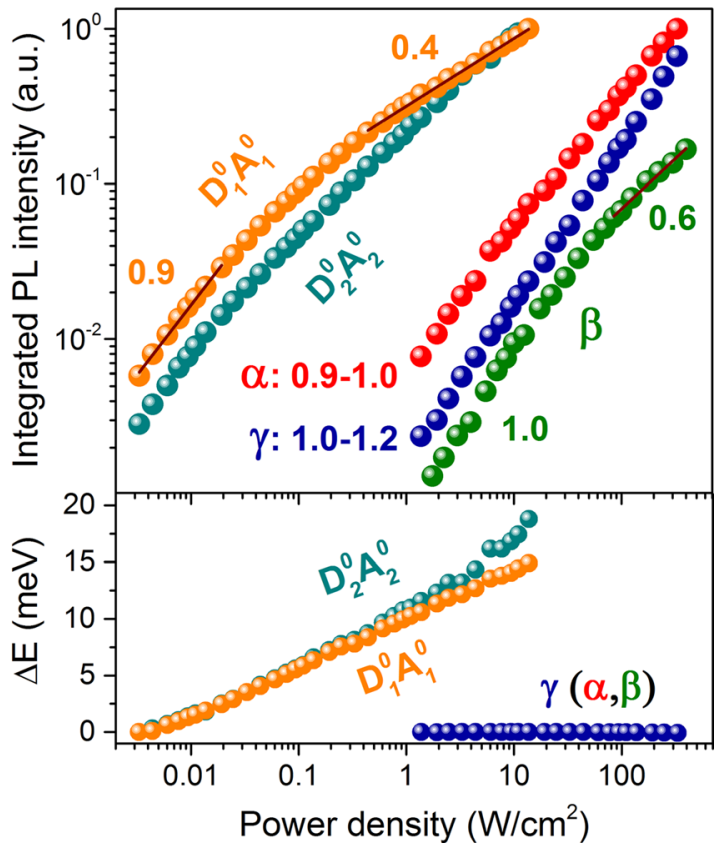

FIG. 2. PL spectra of vertical WZ GaP wires acquired at different excitation powers. At the lowest excitation power, the optical emission is centered at $2.088 \mathrm{eV}$, while the band filling up to $2.253 \mathrm{eV}$ and the $\alpha-\beta-\gamma$ exciton lines are observed at higher excitation powers. Inset: SEM image of patterned GaP nanowires with $200 \mathrm{~nm}$ diameter (tilting angle $80^{\circ}$ ). (b) Integrated PL intensity and energy shift $\Delta \mathrm{E}$ as a function of the excitation power density.

image in supplementary material, Fig. S1. The PL spectrum $(4 \mathrm{~K})$ collected at a low excitation power density of $0.03 \mathrm{~W} /$ $\mathrm{cm}^{2}$ from vertical WZ GaP wires is shown as the black curve in Fig. 2(a). As compared to our previous work, ${ }^{13}$ significant reduction of the emission from deep impurity levels below $2.0 \mathrm{eV}$ is achieved by using a larger size of the patterned area $(2 \mathrm{~mm} \times 2 \mathrm{~mm})$. At the lowest excitation power, we observe two broad emission lines located at $2.115 \mathrm{eV}$ and $2.088 \mathrm{eV}$, which are identified as two different donor-acceptor pair (DAP) recombination, labelled as $\mathrm{D}^{0}{ }_{1} \mathrm{~A}^{0}{ }_{1}$ and $\mathrm{D}_{2}^{0} \mathrm{~A}_{2}^{0}$, together with optical phonon replicas of the $\mathrm{D}^{0}{ }_{2} \mathrm{~A}^{0}{ }_{2}$ peak at $1.996-2.041 \mathrm{eV}$. By increasing the excitation power up to $330 \mathrm{~W} / \mathrm{cm}^{2}$ (blue curve), we observe a slight saturation of the DAP recombination peaks (see Fig. 2(b)) and the rise of a continuum-like emission in the range of $2.180-2.253 \mathrm{eV}$. The continuum emission strongly increases with both increasing excitation density and photon energy. However, a clear drop in the continuum emission is observed at $2.253 \mathrm{eV}$. At high excitation power density, we observe the appearance of three sharp emission lines at 2.140, 2.164, and $2.252 \mathrm{eV}$, labelled as $\alpha, \beta$, and $\gamma$. Across the tens of samples studied, $\alpha-\gamma$ lines are generally (in $\sim 95 \%$ of the wires) observed with similar intensities, while the $\beta$-line is only occasionally (in $\sim 5 \%$ of the wires) present. The emission lines above $2.265 \mathrm{eV}$ are related to the $\mathrm{ZB}$ substrate underneath the as-grown nanowires. ${ }^{34}$ The $\alpha$-line at $2.140 \mathrm{eV}$ shows a FWHM $\sim 220 \mu \mathrm{eV}$. However, multiple peaks with a FWHM lower than $150 \mu \mathrm{eV}$ (beyond the setup resolution limit) are visible when measured on a single wire transferred to a $\mathrm{SiOx} / \mathrm{Si}$ substrate. Similarly, the $\beta$-line at $2.164 \mathrm{eV}$ also shows multiple sharp peaks (see supplementary material,
Fig. S2), which are unresolved in our setup. Instead, the $\gamma$ line at $2.252 \mathrm{eV}$ is observed as a single peak, with a FWHM of $\sim 260 \mu \mathrm{eV}$.

The integrated PL intensity $\left(\mathrm{I}_{\mathrm{PL}}\right)$ of all emission lines as a function of the excitation power density $\left(\mathrm{P}_{\mathrm{EXC}}\right)$ is shown in Fig. 2(b). The slope $m \sim 1$ extracted from fitting the data with the power law $\mathrm{I}_{\mathrm{PL}} \propto \mathrm{P}_{\mathrm{EXC}}{ }^{m}$, together with the independence of the peak energy on the excitation power (see supplementary material, Fig. S3), suggests excitonic recombination for the $\alpha-\beta-\gamma$ peaks. ${ }^{35}$ The power-dependence for the $\mathrm{D}^{0}{ }_{1} \mathrm{~A}^{0}{ }_{1}$ peak in Fig. 2(b) shows saturation of the PL intensity at high excitation power, which is an indication of the emission being related to impurity states. ${ }^{35}$ Furthermore, we observe a strong blue shift $(\sim 5 \mathrm{meV} /$ decade $)$ with increasing excitation power, which is typical for a DAP transition, due to Coulomb interactions between charged donors and acceptors. $^{36,37}$

Additional narrow peaks are visible in the energy range below the $\alpha$-line in Fig. 3(a). Group theory predicts eight phonon normal modes at the $\Gamma$ point: $2 \mathrm{~A}_{1}, 2 \mathrm{E}_{1}, 2 \mathrm{~B}_{1}, 2 \mathrm{E}_{2}$ for the WZ structure (see supplementary material, Fig. S4), 2,16,38 with one $A_{1}$ and one $E_{1}$ acoustic mode, while the remaining six modes are optical. Based on the expected shifts, the sharp peaks in Fig. 3(a) are assigned as acoustical and optical phonon replicas ${ }^{2,16,38}$ of $13 \pm 1 \mathrm{meV}$ (TA) and $48 \pm 2 \mathrm{meV}$ (TO/ LO), respectively. Phonon replicas are often associated with the presence of localized states. ${ }^{39}$ It is important to mention that we observe identical phonon replicas with the $\alpha-\beta$ lines, but not with the $\gamma$-line, indicating that the origin of the $\gamma$-line is distinctly different to the nature of the $\alpha-\beta$ lines. Furthermore, the peaks at $1.996-2.041 \mathrm{eV}$ in the black curve 
(a)

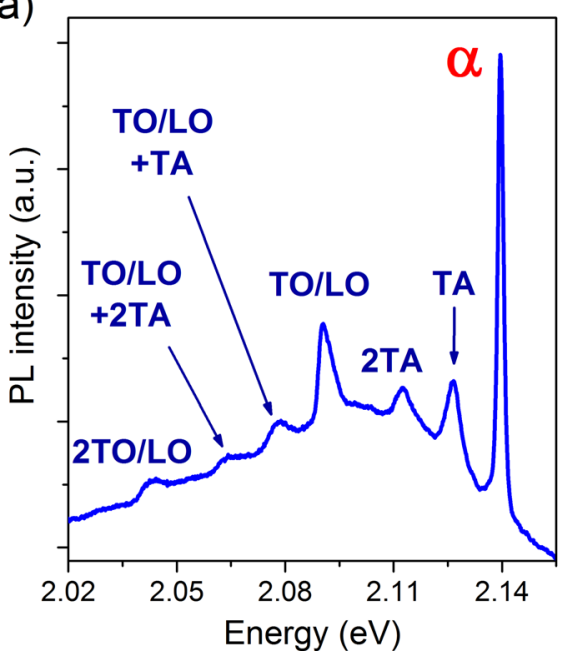

(b)

\begin{tabular}{|c|c|c|}
\hline $\begin{array}{c}\text { Energy } \\
(\mathrm{eV})\end{array}$ & $\begin{array}{c}\Delta \mathrm{E} \\
(\mathrm{meV})\end{array}$ & $\begin{array}{c}\text { Phonon } \\
\text { mode }\end{array}$ \\
\hline 2.140 & - & - \\
\hline 2.126 & 14 & TA \\
\hline 2.113 & 27 & $2 \mathrm{TA}$ \\
\hline 2.091 & 49 & LO/TO \\
\hline 2.078 & 62 & LO/TO+TA \\
\hline 2.064 & 76 & LO/TO+2TA \\
\hline 2.043 & 97 & 2LO/TO \\
\hline
\end{tabular}

FIG. 3. (a) Acoustical (TA) and optical (TO/LO) phonon replicas of the $\alpha$-line. (b) Table of the phonon replicas observed in (a). in Fig. 2(a) are assigned as $\mathrm{LO} / \mathrm{TO}\left(\mathrm{A}_{1}-\mathrm{E}_{1}-\mathrm{E}_{2}\right)$ phonon replicas $(46 \pm 3 \mathrm{meV})^{38}$ of the main $\mathrm{D}^{0}{ }_{2} \mathrm{~A}^{0}{ }_{2}$ peak.

Time-resolved PL measurements as a function of the emission energy are presented in Fig. 4(a). A progressive reduction in lifetime from $\sim 1.0 \mathrm{~ns}$ down to $\sim 0.5 \mathrm{~ns}$ is observed for the band signal with increasing emission energy, while the sharp $\alpha-\beta-\gamma$ emission lines show different features. A long lifetime of $75 \pm 2 \mathrm{~ns}$ is measured for the $\alpha$ line, with a fast decay of $\sim 1.0$ ns obtained for the band signal in the proximity of the $\alpha$-line (see Fig. 4(b)). Thus, when selecting the $\alpha$-line, a low intensity PL signal from the band underneath is still present, which results in a fast decay at the beginning of the red curve in Fig. 4(b). Similar behaviour is observed for the $\beta$-line, with a long lifetime of $43 \pm 2 \mathrm{~ns}$ (see supplementary material, Fig. S2). Instead, the $\gamma$-line shows a different behaviour, with a short lifetime of $0.70 \mathrm{~ns}$ measured at the edge of the continuum-like background signal with $0.55-0.60$ ns decay (see Fig. 4(c)). Furthermore, the lifetime of the $\gamma$-line is almost independent of the excitation power (see inset in Fig. 4(c)), indicating that Auger recombination can be excluded as a non-radiative recombination mechanism for the $\gamma$-line. ${ }^{40}$

(a)

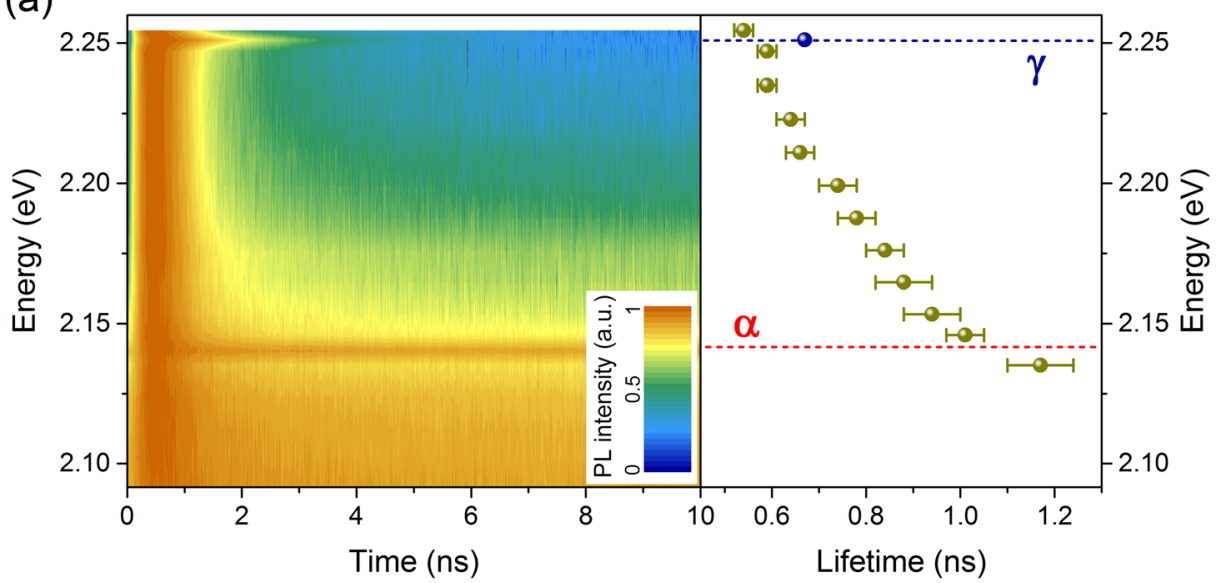

(b)

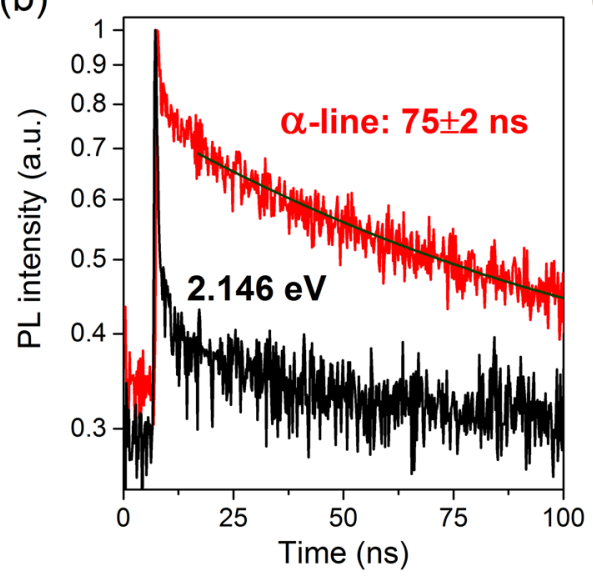

(c)

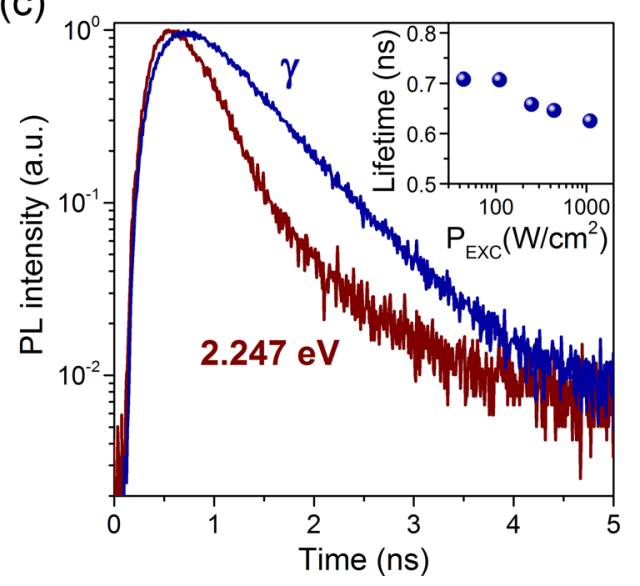

FIG. 4. (a) Time resolved photoluminescence (TRPL) lifetime measurements as a function of the emission energy at $4 \mathrm{~K}$, acquired at $250 \mathrm{~W} / \mathrm{cm}^{2}$ $(10 \mathrm{MHz})$. The emission energies of the $\alpha-\gamma$ lines are indicated by dashed lines. (b) TRPL spectrum of the $\alpha$-line at $4 \mathrm{~K}$, showing a long lifetime of $75 \pm 2 \mathrm{~ns}$, in comparison with the fast decay $\sim 1.0 \mathrm{~ns}$ of the emission at $2.146 \mathrm{eV}$ near the $\alpha$-line. (c) TRPL measurements of the $\gamma$-line at $4 \mathrm{~K}$, showing a short lifetime of $0.70 \mathrm{~ns}$, compared with the $0.55-0.60 \mathrm{~ns}$ of the bandfilling continuum. Inset: Lifetime for the $\gamma$-line as a function of the excitation power density. 
(a)

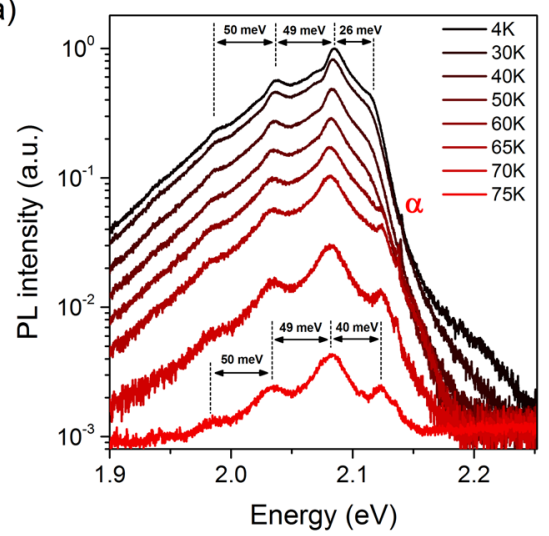

(b)

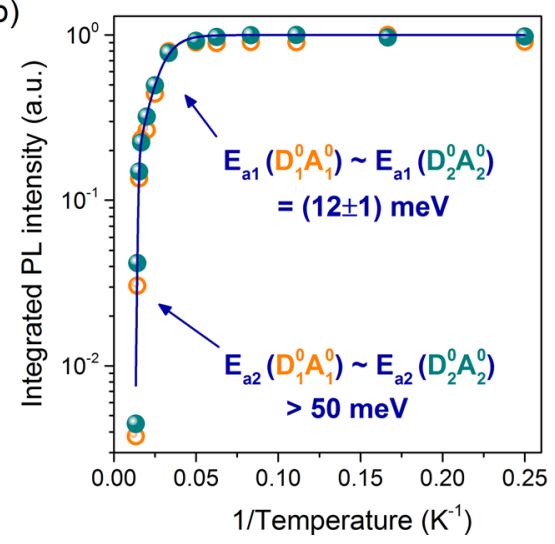

(c)

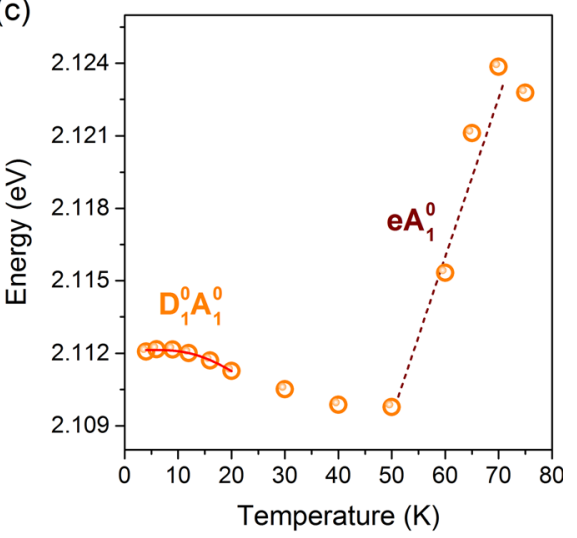

(d)

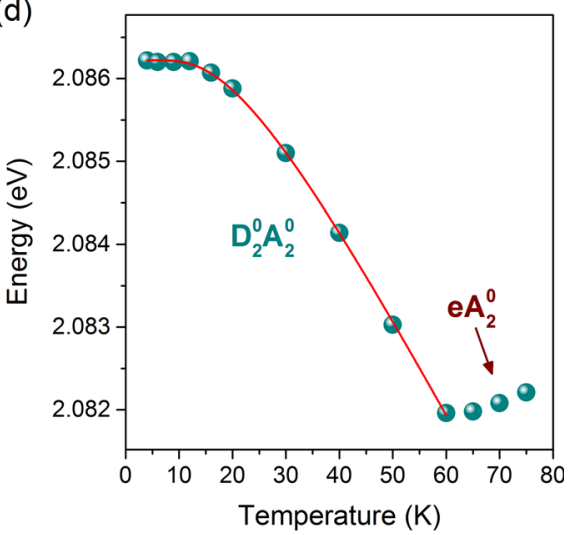

FIG. 5. (a) Temperature dependent PL spectra in the temperature range $4-75 \mathrm{~K}$ acquired in the low power regime of $0.03 \mathrm{~W} / \mathrm{cm}^{2}$. (b) Integrated PL intensity of the $\mathrm{D}^{0}{ }_{1} \mathrm{~A}^{0}{ }_{1}$ and $\mathrm{D}^{0}{ }_{2} \mathrm{~A}^{0}{ }_{2}$ peaks as a function of the reciprocal temperature. The fit of the data using Eq. (1) is shown with a blue solid line. ((c) and (d)) Energy values of the $\mathrm{D}^{0}{ }_{1} \mathrm{~A}^{0}{ }_{1}$ and $\mathrm{D}^{0}{ }_{2} \mathrm{~A}^{0}{ }_{2}$ peaks as a function of temperature. The Varshni fit of the data is shown with a red solid line.
Temperature-dependent PL measurements of vertical wires in the low excitation power regime $\left(0.03 \mathrm{~W} / \mathrm{cm}^{2}\right)$ are shown in Fig. 5. In the range of 4-30 K, a red-shift of the peak energies according to Varshni's law is found for both $\mathrm{D}^{0}{ }_{1} \mathrm{~A}^{0}{ }_{1}$ and $\mathrm{D}^{0}{ }_{2} \mathrm{~A}^{0}{ }_{2}$ peaks (see Figs. 5(c) and 5(d)). ${ }^{41}$ Above $30 \mathrm{~K}$, because of shallow donor ionization, the $\mathrm{D}^{0}{ }_{1} \mathrm{~A}^{0}$ band progressively evolves into a free electron-neutral acceptor band $\left(\mathrm{eA}^{0}{ }_{1}\right) \cdot{ }^{42}$ However, since the precise assignment of acceptor and donor levels is out of the scope of this work, also shallow acceptor ionization into a free hole-neutral donor band $\left(\mathrm{hD}^{0}{ }_{1}\right)$ might take place. Similar ionization behavior is observed also for $\mathrm{D}_{2}^{0} \mathrm{~A}^{0}{ }_{2}$, but at a higher temperature of $60 \mathrm{~K}$, suggesting that higher ionization energies are involved. As a result of this, the energy difference between the two DAP peaks increases from $\sim 26 \mathrm{meV}$ (at $4 \mathrm{~K}$ ) up to $\sim 40 \mathrm{meV}$ (at $75 \mathrm{~K}$ ). In addition, the $\sim 49 \mathrm{meV}$ spacing of the $\mathrm{LO} / \mathrm{TO}$ phonon replicas is weakly dependent on temperature, ${ }^{43}$ as seen for the $1.996-2.041 \mathrm{eV}$ peaks in Fig. 5(a). The integrated PL intensity (I) of the peaks is shown in the Arrhenius plot in Fig. 5(b). The temperature-dependent PL data in Fig. 5(b) can be fitted considering two active nonradiative recombination channels using the equation,

$$
I=1 /\left[1+\mathrm{c}_{1} \cdot \exp \left(-\mathrm{E}_{\mathrm{a} 1} / \mathrm{k} T\right)+\mathrm{c}_{2} \cdot \exp \left(-\mathrm{E}_{\mathrm{a} 2} / \mathrm{k} T\right)\right],
$$

where $c_{1}$ and $c_{2}$ are constants, $E_{a 1}$ and $E_{a 2}$ are the activation energies, and $k$ is the Boltzmann constant. ${ }^{42}$ From fitting the curves in Fig. 5(b) using Eq. (1), we obtain similar activation energies for both DAP peaks, with $E_{a l}\left(\mathrm{D}^{0}{ }_{1} \mathrm{~A}^{0}{ }_{1}\right) \sim$ $E_{a l}\left(\mathrm{D}_{2}^{0}{ }_{2} \mathrm{~A}_{2}^{0}\right)=12 \pm 1 \mathrm{meV}$ and $E_{a 2}\left(\mathrm{D}^{0}{ }_{1} \mathrm{~A}^{0}{ }_{1}\right) \sim E_{a 2}\left(\mathrm{D}^{0}{ }_{2} \mathrm{~A}_{2}^{0}\right)$ $>50 \mathrm{meV}$. From the dependence of the emission energy with temperature in Figs. 5(c) and 5(d), the transition from DAP into a free electron-neutral acceptor band $\left(\mathrm{eA}^{0}\right)$ starts at $\sim 30 \mathrm{~K}$ and $\sim 60 \mathrm{~K}$ for the $\mathrm{D}^{0}{ }_{1} \mathrm{~A}^{0}{ }_{1}$ and $\mathrm{D}^{0}{ }_{2} \mathrm{~A}^{0}{ }_{2}$ peaks, respectively. This would result in $E_{a l}\left(\mathrm{D}^{0}{ }_{1} \mathrm{~A}^{0}{ }_{1}\right)<$ $E_{a l}\left(\mathrm{D}^{0}{ }_{2} \mathrm{~A}_{2}^{0}\right)$, which seems to be in contradiction with the fit in Fig. 5(b). The discrepancy demonstrates how the presence of other non-radiative recombination centers such as surface states, additional deep levels, and increased carrier-phonon scattering with temperature potentially inhibits a precise estimation of the ionization energies of the impurities involved in the $\mathrm{D}^{0}{ }_{1} \mathrm{~A}^{0}{ }_{1}$ and $\mathrm{D}^{0}{ }_{2} \mathrm{~A}^{0}{ }_{2}$ transitions.

Temperature-dependent PL measurements in the high power regime $\left(330 \mathrm{~W} / \mathrm{cm}^{2}\right)$ are plotted in Fig. 6(a). The ZB substrate emission disappears at $30 \mathrm{~K}$ due to the dissociation of excitons bound to nitrogen impurities. ${ }^{2,10}$ With increasing temperature, a reduction in the intensity of band-filling continuum and of the $\alpha-\beta-\gamma$ lines is observed, together with a red-shift of the peak energies according to Varshni's law (see inset in Fig. 6(a)). ${ }^{41}$ The integrated PL intensity of the $\alpha-\beta$ peaks in the Arrhenius plot in Fig. 6(b) is fitted considering one active non-radiative recombination channel for the exciton $^{42}$

$$
I=1 /\left[1+\mathrm{c} \cdot \exp \left(-E_{a} / \mathrm{k} T\right)\right],
$$

while for the fit of the $\gamma$-line, two activation energies are used as in Eq. (1). The values estimated from the fit to the data are listed in Fig. 6(b). In addition, we note that the constants $c_{1}, c_{2}, c$ in Eqs. (1) and (2) are defined as the ratio between the exciton radiative lifetime $\left(\tau_{R}\right)$ and the exciton escape time into a non-radiative recombination center $\left(\tau_{N R}\right){ }^{42,44}$ The density of non-radiative centers is expected to 


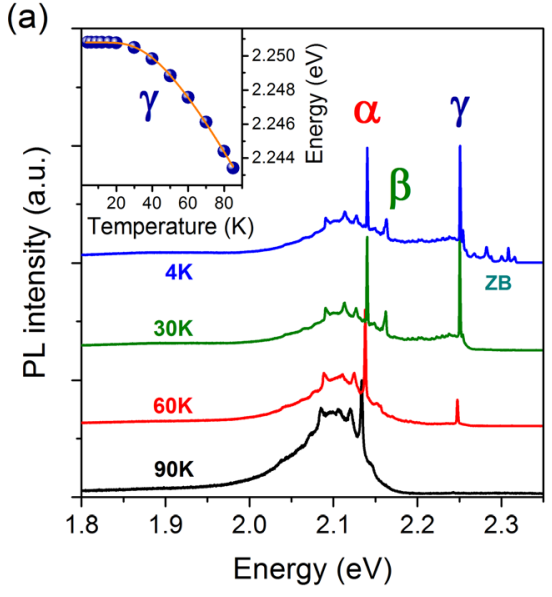

(b)

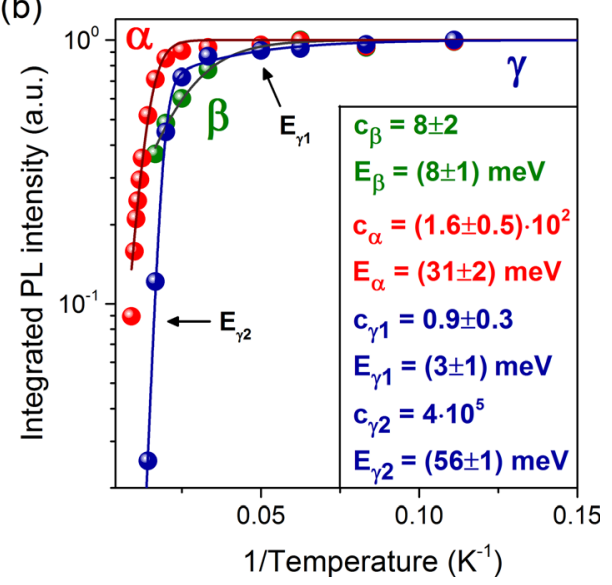

FIG. 6. (a) PL spectra of vertical nanowires acquired in the temperature range $4-100 \mathrm{~K}$ in the high power regime of $330 \mathrm{~W} / \mathrm{cm}^{2}$. Inset: $\gamma$-peak energy values as a function of temperature. (b) Integrated PL intensity of the $\alpha-\beta-\gamma$ peaks as a function of the reciprocal the temperature. be directly proportional to $1 / \tau_{N R}{ }^{44}$ Considering the lifetime measurements in Fig. 4, we estimate $\tau_{N R}{ }^{\alpha}=(0.5 \pm 0.2) \mathrm{ns}$ and $\tau_{N R}^{\beta}=(6 \pm 1) \mathrm{ns}$ for the $\alpha$ - $\beta$ lines, while in the case of the $\gamma$-line, $\tau_{N R}{ }^{\gamma 1}=(0.8 \pm 0.3) \mathrm{ns}$ is obtained in the regime of the first activation energy $E_{\gamma 1}$ and $\tau_{N R}{ }^{\gamma 2}=2 \cdot 10^{-6}$ ns for $E_{\gamma 2}$. The extremely small value of $\tau_{N R}{ }^{\gamma 2}$ indicates that nonradiative recombination channels dominate at temperatures higher than $40 \mathrm{~K}$.

At $100 \mathrm{~K}$, phonon replicas of the $\alpha$-line are still visible in Fig. 7(a), with the additional presence of two phonons at higher energies possibly related to anti-Stokes shifted phonon satellites. ${ }^{45}$ By increasing the excitation power, the intensity of the $\alpha-\gamma$ lines increases (see Fig. 7(a)), together with an increase in the intensity of the continuum up to $\sim 2.32 \mathrm{eV}$ and of the impurity states below $2.0 \mathrm{eV}$. The additional peak observed at $2.31 \mathrm{eV}$ relates to the $\mathrm{ZB}$ substrate underneath the nanowires. When the temperature is raised from $100 \mathrm{~K}$ to $300 \mathrm{~K}$, the $\alpha-\gamma$ excitons dissociate, and the emissions from impurity states and $\mathrm{ZB}$ substrate disappear, leading to room temperature PL emission centered at $2.22 \mathrm{eV}$ originating only from the nanowires (see Fig. 7(b)). This emission has a FWHM in the order of $200 \mathrm{meV}$, covering the amber-green region of wavelengths of the visible spectrum, and may find applications in solid state lighting in the green range of the visible wavelengths. 46

\section{DISCUSSION}

From our experimental investigation, we attribute the recombination at $2.115 \mathrm{eV}$ and $2.088 \mathrm{eV}$ to DAPs which are likely related to the presence of carbon donor and acceptor levels, as estimated by APT. From the estimated donor and acceptor ionization energies, we position the $4 \mathrm{~K}$ direct band gap of WZ GaP above $2.170 \mathrm{eV}$. We discuss the boundexcitonic nature of the $\alpha-\beta-\gamma$ lines based on the combination of PL and TRPL measurements that results in a band gap of $\sim 2.190 \mathrm{eV}$, in agreement with the estimation from the DAP peaks and the theoretical predictions, although they give slightly smaller energies (see Fig. 1(e)). The interaction of the $\alpha-\beta$ excitons with impurity levels allows recombination at larger wave-vector outside the $\Gamma$-point, where an enhancement in the oscillator strength for the $\mathrm{A}\left(\Gamma_{8 \mathrm{c}}-\Gamma_{9 \mathrm{v}}\right)$ band edge is predicted. Furthermore, we attribute the $\gamma$-line to a level resonant in the $\Gamma_{8 \mathrm{c}}$ conduction band, with inter-valley tunneling of electrons from the $\Gamma$ - into the M-minimum for increasing temperature.
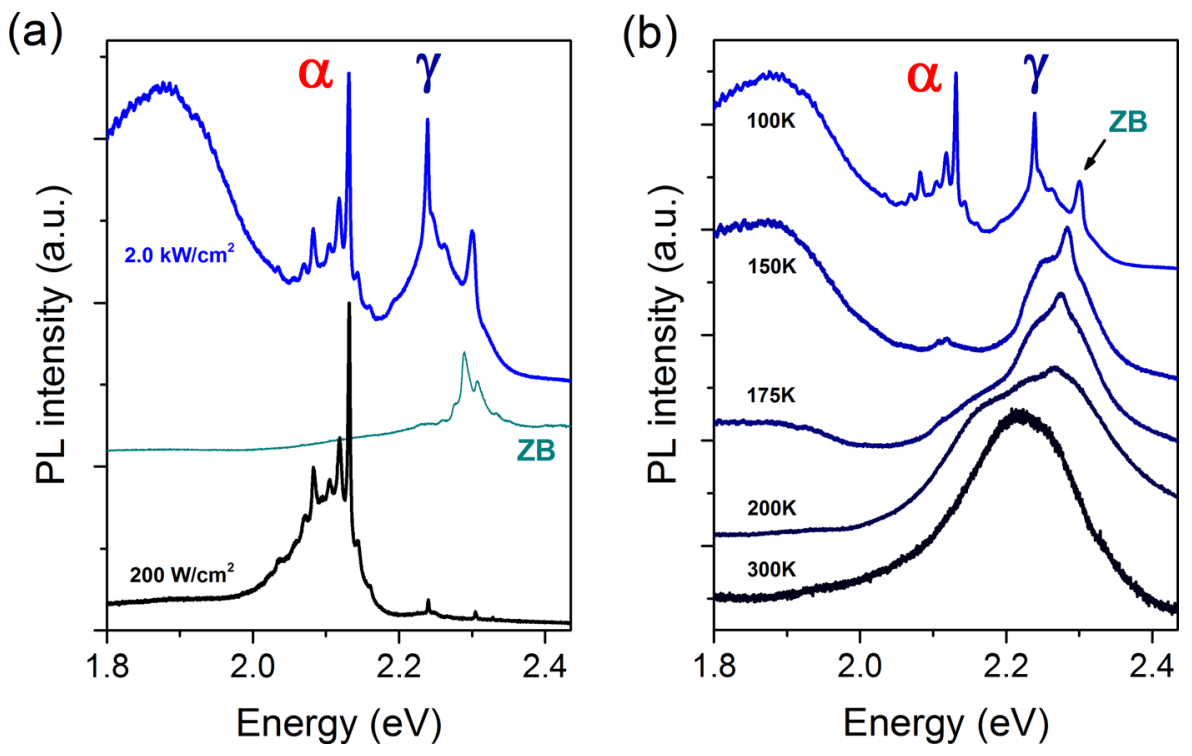

FIG. 7. (a) PL spectra of vertical nanowires at $100 \mathrm{~K}$ for increasing excitation power density. The emission from the $\mathrm{ZB}$ substrate is shown in the aqua curve. (b) Temperature-dependent PL measurements in the temperature range $100-300 \mathrm{~K}$, acquired with an excitation power density of $2.0 \mathrm{~kW} / \mathrm{cm}^{2}$. 


\section{A. DAP centers}

The broad emission lines located at $2.115 \mathrm{eV}\left(\mathrm{D}^{0}{ }_{1} \mathrm{~A}^{0}{ }_{1}\right)$ and $2.088 \mathrm{eV}\left(\mathrm{D}^{0}{ }_{2} \mathrm{~A}^{0}{ }_{2}\right)$ show many features, which are characteristic of a DAP recombination. ${ }^{36,37}$ First, the $2.088 \mathrm{eV}$ emission line is asymmetrically broadened with a FWHM of $\sim 30 \mathrm{meV}$, with the presence of LO/TO phonon replicas. ${ }^{47}$ Second, both $\mathrm{D}^{0}{ }_{1} \mathrm{~A}^{0}{ }_{1}$ and $\mathrm{D}^{0}{ }_{2} \mathrm{~A}^{0}{ }_{2}$ peaks show a strong blue shift of the emission energy $(\sim 5 \mathrm{meV} /$ decade $)$ with increasing excitation power (see Fig. 2(b)) due to Coulomb interactions between charged donors and acceptors. ${ }^{36,37}$ Third, saturation of the PL intensity at high excitation power for the $\mathrm{D}^{0}{ }_{1} \mathrm{~A}^{0}{ }_{1}$ peak indicates emission related to impurity states. ${ }^{35}$ In addition, in the temperature-dependent PL measurements in Fig. 5, we observe impurity ionization with a blue-shift of the emission energy toward either electron-acceptor $\left(\mathrm{eA}^{0}{ }_{1}\right)$ or donor-acceptor $\left(\mathrm{hD}^{0}{ }_{1}\right)$ levels. ${ }^{42}$ These arguments provide compelling evidence that both peaks at $2.115 \mathrm{eV}$ and $2.088 \mathrm{eV}$ (at $4 \mathrm{~K}$ ) can be assigned to DAP recombination.

\section{B. Impurities}

A precise estimation of the impurity concentrations in semiconductor NWs is provided by APT measurements. ${ }^{48,49}$ We mainly find carbon impurities, with a ratio in the concentration of $C_{2}$ complexes and single $C$ atoms larger than 10:1, as listed in Table I (see also Fig. S5 in the supplementary material for more details). The low concentration of aluminum in the nanowires has a negligible effect on the band gap of $\mathrm{WZ} \mathrm{GaP}$. In addition, the presence of oxygen could be related to the sample preparation as well as incorporation in the nanowires.

In $\mathrm{ZB} \mathrm{GaP}$, the impurity levels are related to the indirect $\mathrm{X}$-minimum, and carbon behaves as a shallow acceptor level with a binding energy of $54 \mathrm{meV}$, while shallow donors are in the energy range of $60-110 \mathrm{meV}$ and oxygen is a deep level of $890 \mathrm{meV} .^{50,51}$ In the APT measurements, we do not observe the presence of atoms that can behave as shallow donors. However, donor doping levels lower than $5 \times 10^{16}$, which are not detectable in APT, would still be optically active. In addition, due to the amphoteric nature of carbon in $\mathrm{GaP}$, the $\mathrm{C}$ and $\mathrm{C}_{2}$ impurities could create both donor and acceptor levels in the band gap of WZ GaP, with doping levels in the order of $\sim 6 \times 10^{17}$. Based on the energy values of the DAP peaks and under the assumption that the carbon ionization energy in $\mathrm{WZ} \mathrm{GaP}$ is similar to $\mathrm{ZB} \mathrm{GaP}$ (i.e., $54 \mathrm{meV})$, we estimate the fundamental band gap $\left(\Gamma_{9 \mathrm{v}}-\Gamma_{8 \mathrm{c}}\right)$ of WZ GaP being larger than $2.170 \mathrm{eV}$.

\section{Burstein-Moss bandfilling}

The calculated matrix element for the A transition $\left(\Gamma_{9 \mathrm{v}}-\Gamma_{8 \mathrm{c}}\right)$ shows a very small value at the $\Gamma$-point (see Fig.

TABLE I. Measured concentration of impurities in WZ GaP NWs by APT. Carbon is mostly observed as $\mathrm{C}_{2}$ complexes rather than individual $\mathrm{C}$ atoms. The setup detection limit is approximately $1 \times 10^{-4} \%(1 \mathrm{ppm})$, which is equivalent to a doping level of $5 \times 10^{16} \mathrm{~cm}^{-3}$.

\begin{tabular}{ccccc}
\hline \hline $\mathrm{Ga}(\%)$ & $\mathrm{P}(\%)$ & $\mathrm{C}, \mathrm{C}_{2}(\%)$ & $\mathrm{Al}(\%)$ & $\mathrm{O}(\%)$ \\
\hline $51 \pm 1$ & $50 \pm 1$ & $(1.2 \pm 0.3) \times 10^{-3}$ & $(2.6 \pm 0.3) \times 10^{-3}$ & $(0.6 \pm 0.3) \times 10^{-3}$ \\
\hline \hline
\end{tabular}

1(b)). ${ }^{20}$ Due to the very low radiative recombination probability of the photo-excited carriers in the A band, strong band-filling is expected. As a result, the continuum-like emission between 2.180 and $2.253 \mathrm{eV}$ strongly increases with excitation power (see Fig. 2(a)), suggesting a bandfilling continuum due to the Burstein-Moss effect. ${ }^{37,52,53}$ The increasing magnitude of the continuum emission at higher photon energy is in accordance with the increasing value of the matrix elements at larger $k$-values in Fig. 1(b). In addition, the decrease in lifetime with increasing energy (see Fig. 4(a)) confirms the increase in oscillator strength predicted at larger $k$-values (see Fig. 1(b)). The steep cut-off of the continuum-like emission near the $\gamma$-line suggests a fast recombination channel, which is strongly limiting the bandfilling above it. This is indeed shown by the fast radiative lifetime $(0.70 \mathrm{~ns})$ of the $\gamma$-line. Since band-filling can only be observed above the fundamental band gap, this fact, combined with the presence of DAP peaks at $2.115 \mathrm{eV}$ and $2.088 \mathrm{eV}$, indicates that the fundamental band gap of WZ $\mathrm{GaP}$ is located above the $\alpha-\beta$ lines.

\section{D. $\alpha-\beta-\gamma$ bound-excitons}

Common features of the $\alpha-\beta-\gamma$ lines are the linear scaling of the integrated PL intensity with excitation power density in Fig. 2(b), and the independence of the peak energy position on excitation power (see Fig. S3 in the supplementary material), which resembles direct band gap excitonic recombination. ${ }^{35,37}$ Instead, clear differences are observed in the radiative lifetime (see Fig. 4) and in the presence of the phonon replicas. The $\alpha-\beta$ lines show a long lifetime of $75 \mathrm{~ns}$ and $40 \mathrm{~ns}$ and strong TO/LO-TA phonon replicas, while a short lifetime of $0.70 \mathrm{~ns}$ and no phonon replicas are observed for the $\gamma$-line. Further insight into the nature of the $\alpha-\beta-\gamma$ lines is given by the temperature-dependent PL measurements in Fig. 6. The high activation energy value of $E_{\alpha}=31 \pm 2 \mathrm{meV}$ suggests that the $\alpha$-line is strongly bound to a deep center, which also enhances the intensity of the phonon replicas. ${ }^{51}$ Similarly, the $\beta$-line is weakly bound to a deep center with $E_{\beta}=8 \pm 2 \mathrm{meV}$. The presence of impurity levels that are localized in real space ${ }^{54-56}$ results in delocalization of the electron wave function in $k$-space. This allows an overlap with larger transition matrix elements at larger $k$-values, outside the $\Gamma$-point, which provide a non-zero transition strength for bound excitons and thus a radiative recombination channel for the $\mathrm{A}\left(\Gamma_{9 \mathrm{v}}-\Gamma_{8 \mathrm{c}}\right)$ transition, which would otherwise not emit. The measured $75 \mathrm{~ns}$ and $43 \mathrm{~ns}$ recombination lifetimes for the $\alpha-\beta$ lines confirm the small but non-zero transition strength for the $\mathrm{A}\left(\Gamma_{9 \mathrm{v}}-\Gamma_{8 \mathrm{c}}\right)$ transition.

Concerning the $\gamma$-line, the situation is more complicated. At low temperature, the exciton is weakly bound to deep center $\left(E_{\gamma l}=3 \pm 1 \mathrm{meV}\right)$, which does not induce an enhancement of the intensity of the phonon replicas. The short lifetime for the $\gamma$-line indicates high oscillator strength for this excitonic transition. A possible reason for such a strong increase in the oscillator strength is the significant admixture of $\Gamma_{8 \mathrm{c}}-\Gamma_{7 \mathrm{c}}$ symmetry due to the localized nature of the $\gamma$-line bound-state. ${ }^{17}$ When a transition with (partial) $\Gamma_{7 \mathrm{c}}$ symmetry 
is involved, the high value for the oscillator strength ${ }^{20}$ would result in a short lifetime, as observed in Fig. 4.

The $\gamma$-exciton intensity rapidly decreases above $40 \mathrm{~K}$. The thermal energy at these temperatures is much lower than the calculated exciton binding energy of $\sim 17 \mathrm{meV}$ (see Fig. S7 in the supplementary material). Thus, the steep decrease in PL intensity with an activation energy of $E_{\gamma 2}=56 \pm 1 \mathrm{meV}$ indicates that another non-radiative recombination channel becomes dominant at high temperature, such as inter-valley tunnelling of electrons into the indirect M-minimum in the conduction band, ${ }^{57,58}$ as it will be discussed in Section IV E.

We occasionally observe sharp crystal phase quantum well (CPQW) emission lines at energies in between the $\alpha$ and $\gamma$-lines, which are associated with the presence of stacking faults in the WZ nanowires. ${ }^{59}$ However, the CPQW emission energy is determined by the length of the ZB barrier, while the $\alpha-\gamma$ lines are always observed at the same energy in all samples studied, as it will be discussed more in detail in a separate publication. ${ }^{59}$

\section{E. Optical band structure}

We now build up the electronic band diagram of WZ $\mathrm{GaP}$ from spectroscopic data based on the combination of peak energies and activation energies estimated in the PL measurements. From the DAP emission in the low power regime, we estimate a band gap $E_{\mathrm{G}}{ }^{\mathrm{DAP}}$ higher than $2.170 \mathrm{eV}$. Next, we consider $\alpha-\beta-\gamma$ peaks as bound excitons, and the transition level $E_{0}^{j}$ is estimated as $E^{\mathrm{PL}}{ }_{j}=E^{j}{ }_{0}-E_{B X}^{j}-E_{X}$ (Eq. (3)), where $\mathrm{j}=\alpha, \beta, \gamma, E^{\mathrm{PL}}{ }_{j}$ is the peak energy in the PL spectrum, $E_{B X}^{j}$ is the exciton binding energy to an impurity (i.e., $E_{A}$ in Fig. 6), and $E_{X}$ is the exciton binding energy. For the exciton binding energy, we use the predicted value $E_{\mathrm{X}}=E_{n}{ }^{\text {,Theory }}=17 \mathrm{meV}$ (see Fig. S7 in the supplementary material). We estimate $E^{\alpha}{ }_{0}=2.188 \pm 0.005 \mathrm{eV}, E^{\beta}{ }_{0}=2.189$ $\pm 0.005 \mathrm{eV}$, and $E_{0}^{\gamma}=2.272 \pm 0.005 \mathrm{eV}$, as shown in Fig. 8(a). The values $E^{\alpha}{ }_{0}, E^{\beta}{ }_{0}$ are in agreement with $E_{G}{ }^{D A P}$, indicating a direct band gap for $\mathrm{WZ}$ GaP of $E_{G}$ (WZ) $\sim 2.190 \mathrm{eV}$. Interestingly, the value $E_{0}^{\gamma}$ is larger than $E_{G}$ (WZ) by $\sim 80 \mathrm{meV}$. Polarization and pressure-dependent PL measurements on WZ GaP nanowires strongly suggest that the $\gamma$-transition is neither related to the indirect M-minimum nor to the light hole or split-off band. ${ }^{17}$ Instead, significant admixture of $\Gamma_{7 \mathrm{c}}$-symmetry due to high localization of the associated state and a low density of states in the conduction band ${ }^{60}$ promotes an efficient transition from a resonant level to the $\Gamma_{9 \mathrm{v}}$ valence band. Thus, the $\gamma$-line is attributed to a level resonant in the $\Gamma_{8 \mathrm{c}}$ conduction band. ${ }^{17,60}$

A possible non-radiative recombination channel that dominates the $\gamma$-line recombination at high temperature (see Fig. 6) is the inter-valley tunnelling of electrons into the indirect $\mathrm{M}$-minimum in the conduction band. ${ }^{57,58}$ In the indirect band gap semiconductors like germanium, the L-minimum in the conduction band lies below the $\Gamma$-minimum, with an energy difference of $136 \mathrm{meV} .{ }^{57,58}$ Under an optical excitation, when the temperature of the sample is increased, a higher number of electrons are thermally excited from the (indirect) L-minimum into the (direct) $\Gamma$-minimum, leading to a strong increase in PL intensity of the $\Gamma$-point transition. ${ }^{57,58}$ In the case of $\mathrm{WZ} \mathrm{GaP,} \mathrm{the} \mathrm{indirect} \mathrm{M-minimum} \mathrm{is}$ predicted at $\Delta E_{\Gamma-\mathrm{M}}{ }^{\mathrm{DFT}}=110 \mathrm{meV}$ above the $\Gamma$-minimum. ${ }^{20}$ In the high power regime at which the $\alpha-\beta-\gamma$ peaks are observed, the Fermi level increases and a Burstein-Moss band-filling is observed. At temperatures below $40 \mathrm{~K}$, the $\gamma$-exciton is bound to a donor-line center, while above this temperature thermal excitation of the carriers from $\Gamma$ - into M-minimum is observed, as shown in Fig. 8(b). This process
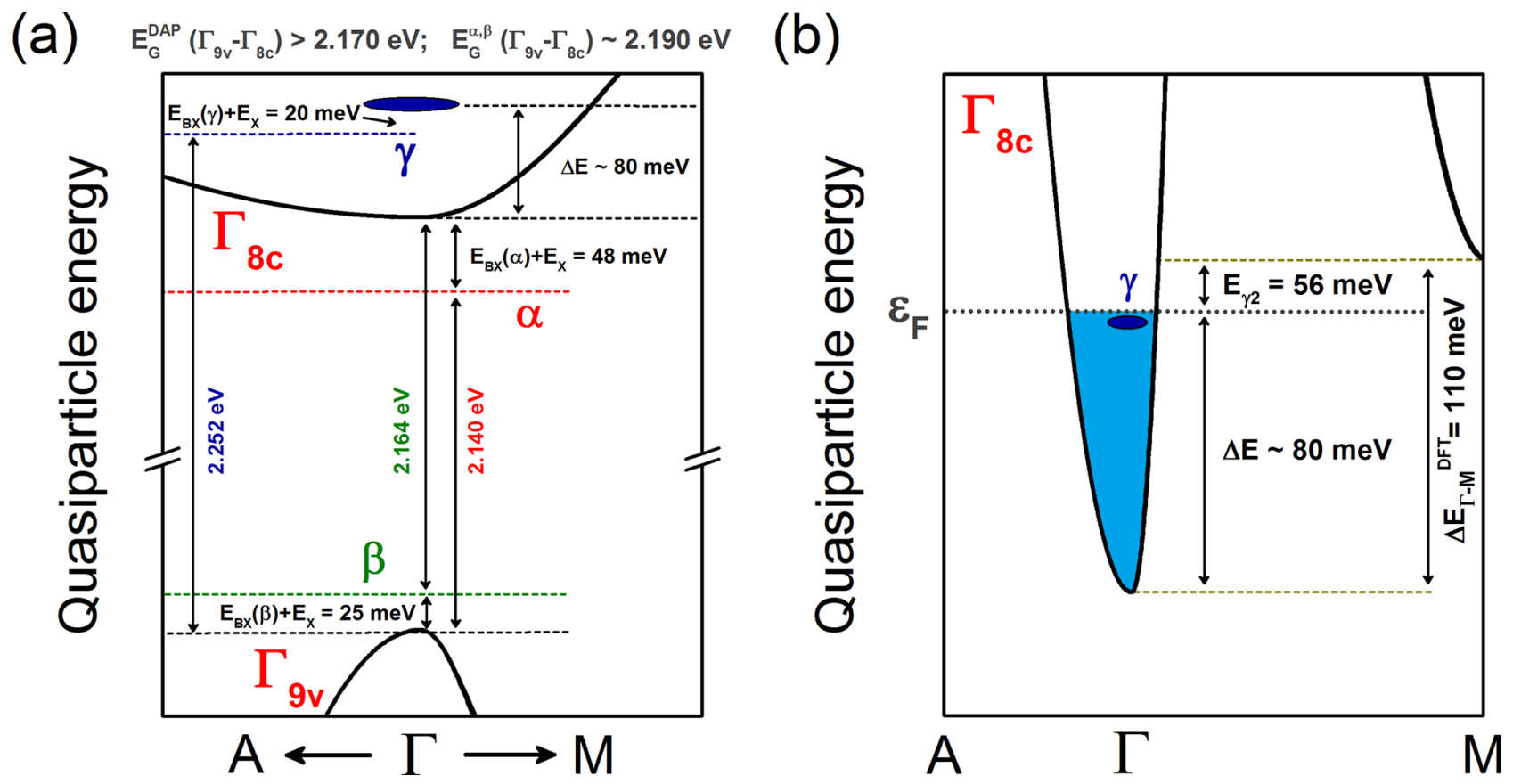

FIG. 8. (a) Predicted direct band structure of WZ GaP with the energy position of deep centers related $\alpha$ - $\beta$ - $\gamma$ exciton transitions indicated by dashed lines and arrows. The donor-like center associated with the $\gamma$-line above the conduction band is indicated with a solid elliptical shape. (b) Predicted conduction band of WZ GaP at high excitation power, showing the inter-valley tunneling of electrons from $\Gamma$ - into M-minimum. 
takes place with the activation energy $E_{\gamma^{2}}=56 \pm 1 \mathrm{meV}$ as estimated in Fig. 6(b), which might very well be the difference between the Fermi level and the M-minimum. ${ }^{57,58}$ By considering the Fermi level close to the $\gamma$-line in the high excitation regime, ${ }^{53}$ we calculate the energy difference between the $\Gamma$ - and M-minimum $\Delta E_{\Gamma-\mathrm{M}}{ }^{\mathrm{PL}}=\Delta E+E_{\gamma 2}$ $\sim 135 \mathrm{meV}$, which is in good agreement with the theoretical value $\Delta E_{\Gamma-\mathrm{M}}{ }^{\mathrm{DFT}}=110 \mathrm{meV} \cdot{ }^{20}$ Furthermore, the extremely small value of $\tau_{N R}{ }^{\gamma 2}$ in Fig. 6(b) is in agreement with a nonradiative transition dominating the recombination for increasing temperature, such as the transition to the indirect (M) minimum with higher density of states compared to the $\Gamma$-minimum. ${ }^{61}$

\section{CONCLUSION}

In conclusion, we performed a detailed study on the optical properties of WZ GaP by performing power- and temperature-dependent PL measurements, combined with TRPL and APT measurements. We showed donor-acceptor pair (DAP) recombination peaks at $2.115 \mathrm{eV}\left(\mathrm{D}^{0}{ }_{1} \mathrm{~A}^{0}{ }_{1}\right)$ and $2.088 \mathrm{eV}\left(\mathrm{D}_{2}^{0} \mathrm{~A}_{2}^{0}\right)$, and Burstein-Moss band-filling continuum between 2.180 and $2.253 \mathrm{eV}$, indicating a $4 \mathrm{~K}$ direct band gap of WZ GaP above $2.170 \mathrm{eV}$. Sharp $\alpha-\beta$ emission lines are observed at $2.140-2.164 \mathrm{eV}$, with both TA and LO/ TO phonon sidebands and long lifetimes of $75 \mathrm{~ns}$ and $43 \mathrm{~ns}$, respectively, while the narrow $\gamma$-line at $2.252 \mathrm{eV}$ shows no phonon replicas and a short lifetime of $0.70 \mathrm{~ns}$. At $4 \mathrm{~K}$, the $\alpha$ $\beta$ lines behave as bound-excitons and a band gap of $\sim 2.190 \mathrm{eV}$ is obtained, in agreement with the estimation from the DAP peaks. The localization of the $\alpha-\beta$ excitons at deep centers allows recombination at larger wave-vector outside the $\Gamma$-point where an enhancement in oscillator strength for the $\mathrm{A}\left(\Gamma_{8 \mathrm{c}}-\Gamma_{9 \mathrm{v}}\right)$ band edge is predicted. In addition, the $\gamma$-line is assigned to a bound-exciton that is $\sim 80 \mathrm{meV}$ above the $\Gamma_{8 \mathrm{C}}$ conduction band edge and acquires oscillator strength by significant $\Gamma_{8 \mathrm{c}}-\Gamma_{7 \mathrm{c}}$ symmetry admixture. Furthermore, possible inter-valley tunneling of electrons from the $\Gamma$ - into the $\mathrm{M}$-minimum for increasing temperature results in a decrease in the intensity of the $\gamma$-line.

\section{SUPPLEMENTARY MATERIAL}

See supplementary material for additional TEM, APT, and optical measurements.

\section{ACKNOWLEDGMENTS}

The authors thank P. J. van Veldhoven for the technical support with the MOVPE reactor, M. A. Verheijen for the TEM investigation, and A. Silov, T. T. T. Vu, D. van Dam, and L. Gagliano for the fruitful discussion. We thank the Dutch Organization for Scientific Research (NWO-VICI 700.10.441). NWO is also acknowledged for funding the Atom Probe facility. J. Greil acknowledges funding by the Austrian Science Fund (FWF): J3540-N30.

${ }^{1}$ T. N. Morgan, Phys. Rev. Lett. 21, 819 (1968).

${ }^{2}$ P. Dean and D. Thomas, Phys. Rev. 150, 690 (1966).

${ }^{3}$ A. Belabbes, C. Panse, J. Furthmüller, and F. Bechstedt, Phys. Rev. B 86, 075208 (2012).
${ }^{4}$ A. Belabbes, J. Furthmüller, and F. Bechstedt, Phys. Rev. B 87, 035305 (2013).

${ }^{5}$ A. De and C. E. Pryor, Phys. Rev. B 81, 155210 (2010).

${ }^{6}$ H. J. Joyce, J. Wong-Leung, Q. Gao, H. H. Tan, and C. Jagadish, Nano Lett. 10, 908 (2010)

${ }^{7}$ S. Assali, L. Gagliano, D. S. Oliveira, M. A. Verheijen, L.-F. Feiner, S. R. Plissard, and E. P. A. M. Bakkers, Nano Lett. 15, 8062 (2015).

${ }^{8}$ N. Vainorius, S. Lehmann, D. Jacobsson, L. Samuelson, K. A. Dick, and M.-E. Pistol, Nano Lett. 15, 2652 (2015).

${ }^{9}$ H. Shtrikman, R. Popovitz-Biro, A. Kretinin, L. Houben, M. Heiblum, M. Bukala, M. Galicka, R. Buczko, and P. Kacman, Nano Lett. 9, 1506 (2009).

${ }^{10}$ C.-Y. Yeh, S.-H. Wei, and A. Zunger, Phys. Rev. B 50, 2715 (1994).

${ }^{11}$ D. Spirkoska, A. L. Efros, W. R. L. Lambrecht, T. Cheiwchanchamnangij, A. Fontcuberta i Morral, and G. Abstreiter, Phys. Rev. B 85, 045309 (2012).

${ }^{12}$ B. Loitsch, J. Winnerl, G. Grimaldi, J. Wierzbowski, D. Rudolph, S. Morkötter, M. Döblinger, G. Abstreiter, G. Koblmüller, and J. J. Finley, Nano Lett. 15, 7544 (2015).

${ }^{13}$ S. Assali, I. Zardo, S. Plissard, D. Kriegner, M. A. Verheijen, G. Bauer, A. Meijerink, A. Belabbes, F. Bechstedt, J. E. M. Haverkort, and E. P. A. M. Bakkers, Nano Lett. 13, 1559 (2013).

${ }^{14}$ A. Berg, S. Lehmann, N. Vainorius, A. Gustafsson, M.-E. Pistol, L. R. Wallenberg, L. Samuelson, and M. T. Borgström, J. Cryst. Growth 386, 47 (2014).

${ }^{15}$ A. Berg, K. Mergenthaler, M. Ek, M.-E. Pistol, L. Reine Wallenberg, and M. T. Borgström, Nanotechnology 25, 505601 (2014).

${ }^{16}$ J. K. Panda, A. Roy, M. Gemmi, E. Husanu, A. Li, D. Ercolani, and L. Sorba, Appl. Phys. Lett. 103, 023108 (2013).

${ }^{17}$ J. Greil, S. Assali, Y. Isono, A. Belabbes, F. Bechstedt, F. O. Valega Mackenzie, A. Y. Silov, E. P. A. M. Bakkers, and J. E. M. Haverkort, Nano Lett. 16(6), 3703 (2016).

${ }^{18}$ S. Assali, D. van Dam, J. E. M. Haverkort, and E. P. A. M. Bakkers, Appl. Phys. Lett. 108, 173101 (2016).

${ }^{19}$ A. Standing, S. Assali, L. Gao, M. A. Verheijen, D. van Dam, Y. Cui, P. H. L. Notten, J. E. M. Haverkort, and E. P. A. M. Bakkers, Nat. Commun. 6, 7824 (2015).

${ }^{20}$ F. Bechstedt and A. Belabbes, J. Phys. Condens. Matter 25, 273201 (2013).

${ }^{21}$ P. Tronc, Y. E. Kitaev, G. Wang, M. F. Limonov, A. G. Panfilov, and G. Neu, Phys. Status Solidi 216, 599 (1999).

${ }^{22}$ P. Tronc, Y. E. Kitaev, G. Wang, and M. F. Limonov, Phys. Status Solidi 210, 471 (1998)

${ }^{23}$ F. Bechstedt, Many-Body Approach to Electronic Excitations (SpringerVerlag, Berlin/Heidelberg, 2015).

${ }^{24}$ P. J. Dean, Phys. Rev. 168, 889 (1968).

${ }^{25}$ P. Hohenberg and W. Kohn, Phys. Rev. 136, B864 (1964).

${ }^{26}$ W. Kohn and L. J. Sham, Phys. Rev. 140, A1133 (1965).

${ }^{27}$ G. Kresse and J. Furthmüller, Phys. Rev. B 54, 11169 (1996).

${ }^{28}$ G. Kresse and J. Furthmüller, Comput. Mater. Sci. 6, 15 (1996).

${ }^{29}$ J. P. Perdew and A. Zunger, Phys. Rev. B 23, 5048 (1981).

${ }^{30}$ G. Kresse and D. Joubert, Phys. Rev. B 59, 1758 (1999).

${ }^{31}$ L. G. Ferreira, M. Marques, and L. K. Teles, Phys. Rev. B 78, 125116 (2008).

${ }^{32}$ J. C. Slater and K. H. Johnson, Phys. Rev. B 5, 844 (1972).

${ }^{33}$ J. Leite and L. Ferreira, Phys. Rev. A 3, 1224 (1971).

${ }^{34}$ P. J. Dean, Phys. Rev. 157, 655 (1967).

${ }^{35}$ T. Schmidt, K. Lischka, and W. Zulehner, Phys. Rev. B 45, 8989 (1992).

${ }^{36}$ A. Ben Slimane, A. Najar, R. Elafandy, D. P. San-Román-Alerigi, D. Anjum, T. K. Ng, and B. S. Ooi, Nanoscale Res. Lett. 8, 342 (2013).

${ }^{37}$ U. Kaufmann, M. Kunzer, M. Maier, H. Obloh, A. Ramakrishnan, B. Santic, and P. Schlotter, Appl. Phys. Lett. 72, 1326 (1998).

${ }^{38}$ B. Pödör, Phys. Status Solidi 120, 207 (1983).

${ }^{39}$ M. Smith, J. Y. Lin, X. Jiang, A. Khan, Q. Chen, A. Salvador, A. Botchkarev, W. Kim, and H. Morkoc, Appl. Phys. Lett. 70, 2882 (1997).

${ }^{40}$ W. Guo, M. Zhang, P. Bhattacharya, and J. Heo, Nano Lett. 11, 1434 (2011)

${ }^{41}$ P. Y. Varshni, Physica 34, 149 (1967).

${ }^{42}$ M. Leroux, N. Grandjean, B. Beaumont, G. Nataf, F. Semond, J. Massies, and P. Gibart, J. Appl. Phys. 86, 3721 (1999).

${ }^{43}$ F. Gervais and B. Piriou, Phys. Rev. B 10, 1642 (1974).

${ }^{44}$ S. A. Lourenc, o, I. F. L. Dias, L. C. Poc sas, J. L. Duarte, J. B. B. de Oliveira, and J. C. Harmand, J. Appl. Phys. 93, 4475 (2003).

${ }^{45}$ K. Luchner and H. Micklitz, J. Lumin. 18-19, 882 (1979).

${ }^{46}$ S. Nakamura, MRS Bull. 34, 101 (2009).

${ }^{47}$ C. J. Chou and G. F. Neumark, Appl. Phys. Lett. 65, 761 (1994). 
${ }^{48}$ S. Koelling, N. Innocenti, A. Schulze, M. Gilbert, A. K. Kambham, and W. Vandervorst, J. Appl. Phys. 109, 104909 (2011).

${ }^{49}$ S. Koelling, A. Li, A. Cavalli, S. Assali, D. Car, S. Gazibegovic, E. P. A. M. Bakkers, and P. M. Koenraad (unpublished)

${ }^{50}$ See http://www.ioffe.rssi.ru/SVA/NSM/Semicond/ for the impurity levels ionization energies.

${ }^{51}$ P. J. Dean, Prog. Solid State Chem. 8, 1 (1973).

${ }^{52} \mathrm{M}$. Grundmann, The Physics of Semiconductors (Springer, 2006).

${ }^{53}$ E. Burstein, Phys. Rev. 93, 632 (1954).

${ }^{54}$ B. K. Meyer, H. Alves, D. M. Hofmann, W. Kriegseis, D. Forster, F. Bertram, J. Christen, A. Hoffmann, M. Straßburg, M. Dworzak, U. Haboeck, and A. V. Rodina, Phys. Status Solidi 241, 231 (2004).

${ }^{55}$ B. Monemar, P. P. Paskov, J. P. Bergman, G. Pozina, A. A. Toropov, T. V. Shubina, T. Malinauskas, and A. Usui, Phys. Rev. B 82, 235202 (2010).
${ }^{56}$ L. Wang and N. C. Giles, J. Appl. Phys. 94, 973 (2003).

${ }^{57}$ X. Sun, J. Liu, L. C. Kimerling, and J. Michel, Appl. Phys. Lett. 95, 011911 (2009).

${ }^{58}$ F. Pezzoli, F. Isa, G. Isella, C. V. Falub, T. Kreiliger, M. Salvalaglio, R. Bergamaschini, E. Grilli, M. Guzzi, H. von Känel, and L. Miglio, Phys. Rev. Appl. 1, 44005 (2014).

${ }^{59}$ S. Assali, J. Lähnemann, T. T. T. Vu, K. Johns, L. Gagliano, M. A. Verheijen, J. E. M. Haverkort, and E. P. A. M. Bakkers (unpublished).

${ }^{60}$ D. R. Scifres, H. M. Macksey, N. J. Holonyak, R. D. Dupuis, G. W. Zack, C. B. Duke, G. G. Kleiman, and A. B. Kunz, Phys. Rev. B 5, 2206 (1972).

${ }^{61}$ S. Wirths, R. Geiger, N. V. Den Driesch, G. Mussler, T. Stoica, S. Mantl, Z. Ikonic, M. Luysberg, S. Chiussi, J. M. Hartmann, H. Sigg, J. Faist, D. Buca, and D. Grützmacher, Nat. Photonics 9, 88 (2015). 